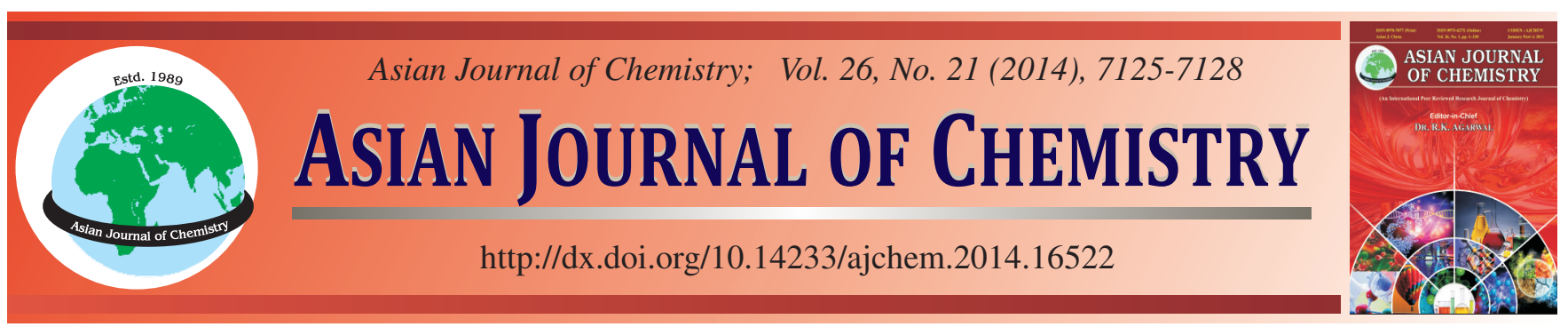

\title{
A Novel Synthesis of Carboxylic Acid Oxime Esters Catalyzed by 2-(1H-Benzotriazole-1-yl)-1,1,3,3-tetramethyluronium Tetrafluoroborate
}

Wei Shi, Jiming Zhang ${ }^{*}$, Qin Zhou, Xinguan Wang, Xiaowei Qi, Lianyong Guo, Wanyong Ma and Jianhua Zhou

School of Chemistry and Pharmaceutical Engineering, Qilu University of Technology, Jinan 250353, P.R. China

*Corresponding author: E-mail: zhangjiming@gmail.com; slzshiwei@163.com

We describe a facile synthesis of carboxylic acid oxime esters in acetonitrile from the corresponding carboxylic acids and oximes catalyzed by 2-(1H-benzotriazole-1-yl)-1,1,3,3-tetramethyluronium tetrafluoroborate in the presence of triethylamine at room temperature under mild conditions.

Keywords: Oxime ester, Carboxylic acid, 2-(1H-Benzotriazole-1-yl)-1,1,3,3-tetramethyluronium tetrafluoroborate, Oxime esterification. ᄂ - - - - - - - - - - - - - - - - - - - - - - - - - - - - - -

\section{INTRODUCTION}

Oxime esters and their derivatives are valuable products or intermediates in the synthesis of medicinal compounds ${ }^{1,2}$ and bioactive agents ${ }^{3-6}$. They are also important starting materials in the preparation of photosensitive compositions s.8. $^{7,}$ Oxime esters are generally synthesized from the corresponding oximes reacting with the high sterically hindered acyl halide ${ }^{9-11}$, anhydride ${ }^{12}$, benzoyl peroxide ${ }^{13}$ and potassium methanecarbotelluroate ${ }^{14}$. However, these methods have not been entirely satisfactory owing to such drawbacks as low yield, long reaction time, difficulty in obtaining reagents, high temperature media, harsh reaction conditions, tedious work-up procedures and so on.

Generally the formation of carboxylic acid oxime esters requires activation of the related carboxyl groups. In the last decade, many peptide coupling reagents have been designed, synthesized and commonly used in peptide synthesis for activating carboxyl groups, such as DCC/DMAP ${ }^{15}$, phosphorus oxychloride ${ }^{16}, \mathrm{COMU}^{17}$, etc. The most successful approaches known today involve activation of the carboxylic group with uronium/guanidinium salts. The most popular members of this family are coupling reagents based on benzotriazole derivatives such as HOBt or HOAt, both of which are also commonly used as additives in carbodiimide-mediated peptide coupling. Therefore, we wish to choose a coupling reagent in this family with a high performance-to-cost ratio.

A uronium-based peptide coupling agent, namely, 2-(1Hbenzotriazole-1-yl)-1,1,3,3-tetramethyluronium tetrafluoroborate is one of the most common coupling reagents and additives used in peptide chemistry ${ }^{18-20} .2-(1 H$-Benzotriazole-1-yl)- 1,1,3,3-tetramethyluronium tetrafluoroborate is a mild and effective coupling reagent that has some favorable characteristics from an industrial or environmental standpoint, such as high efficiency, need for only stochiometric quantities and solubility in currently used solvents. Herein, we report that 2-(1H-benzotriazole-1-yl)-1,1,3,3-tetramethyluronium tetrafluoroborate can be used as a reactive coupling reagent to promote carboxylic acid oxime ester formation at room temperature (Scheme-I).

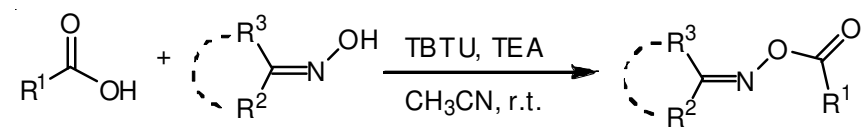

1 3

Scheme-I: Synthesis of carboxylic acid oxime esters

\section{EXPERIMENTAL}

All reagents and solvents were purchased from commercial sources, unless otherwise noted and were used without purification. Melting points were determined on a SGW X-4 melting-point apparatus with microscope and were uncorrected. Infrared spectra were recorded on an IRPrestige-21 FTIR spectrophotometer ( $\mathrm{KBr}) .{ }^{1} \mathrm{H}$ and ${ }^{13} \mathrm{C}$ NMR spectra were recorded on a Bruker Advance-II spectrometer at 400 and 100 $\mathrm{MHz}$ with TMS and referenced to DMSO- $d_{6} ; \delta$ values are reported in ppm and $J$ values in $\mathrm{Hz}$.

General procedure for the synthesis of compound 3a: To a solution of benzoic acid (244 mg, $2 \mathrm{mmol})$ and 2-(1Hbenzotriazole-1-yl)-1,1,3,3-tetramethyluronium tetrafluoroborate $(706 \mathrm{mg}, 2.2 \mathrm{mmol})$ in $40 \mathrm{~mL}$ acetonitrile, triethylamine $(0.56 \mathrm{~mL}, 4 \mathrm{mmol})$ was added and the mixture stirred at room 
temperature for $0.5 \mathrm{~h}$. Then, benzophenone oxime (394 mg, 2 $\mathrm{mmol}$ ) was added and the reaction mixture stirred at room temperature for $0.5 \mathrm{~h}$. The progress of the reaction was monitored by TLC (petroleum ether/ethyl acetate 3:1). After completion of the reaction, the reaction mixture was washed with distilled water and extracted with $\mathrm{CH}_{2} \mathrm{Cl}_{2}(3 \times 10 \mathrm{~mL})$. The combined organic layers were then washed with saturated brine solution $(5 \mathrm{~mL})$, dried over anhydrous magnesium sulfate, filtered, concentrated in vacuo and purified by silica gel column chromatography to obtain pure product.

Compound 3a: White solid, m.p. $100-102{ }^{\circ} \mathrm{C}$ (lit. ${ }^{20}$ $\left.101{ }^{\circ} \mathrm{C}\right) .{ }^{1} \mathrm{H}$ NMR $\left(400 \mathrm{MHz}, \mathrm{DMSO}-d_{6}\right): \delta \mathrm{H} 7.69$ (d, 2H, $J$ $7.6 \mathrm{~Hz}, \mathrm{Ar}-\mathrm{H}), 7.64$ (t, 1H, J 7.4 Hz, Ar-H), 7.57 (d, 3H, J 7.1 $\mathrm{Hz}, \mathrm{Ar}-\mathrm{H}), 7.46-7.52$ ( m, $12 \mathrm{H}, \mathrm{Ar}-\mathrm{H}) .{ }^{13} \mathrm{C} \mathrm{NMR}(100 \mathrm{MHz}$, DMSO- $\left.d_{6}\right)$ : $\delta 165.5,163.8,134.6,133.2,132.8,131.0,129.7$, 129.6, 129.1, 128.8, 128.7, 128.4, 128.2. FT-IR (KBr, $v_{\max }$, $\mathrm{cm}^{-1}$ ): 3061, 1740, 1446, 1244, 1087, 982, 868, 702.

Compound 3b: White solid, m.p. $98-100{ }^{\circ} \mathrm{C}$ (lit. ${ }^{20} 98-$ $\left.100{ }^{\circ} \mathrm{C}\right) .{ }^{1} \mathrm{H}$ NMR $\left(400 \mathrm{MHz}, \mathrm{DMSO}-d_{6}\right): \delta \mathrm{H} 8.09$ (d, 2H, $J$ $7.5 \mathrm{~Hz}, \mathrm{Ar}-\mathrm{H}), 7.83$ (d, 2H, J 6.9 Hz, Ar-H), 7.73 (t, 1H, J 7.4 $\mathrm{Hz}, \mathrm{Ar}-\mathrm{H}), 7.60$ (t, 2H, J 7.6Hz, Ar-H), 7.49-7.56 (m, 3H, $\mathrm{Ar}-\mathrm{H}), 2.52\left(\mathrm{~s}, 3 \mathrm{H}, \mathrm{CH}_{3}\right) .{ }^{13} \mathrm{C}$ NMR $\left(100 \mathrm{MHz}, \mathrm{DMSO}-d_{6}\right): \delta$ 164.2, 164.1, 134.7, 133.8, 131.1, 130.2, 130.1, 129.3, 129.0, 128.8, 127.6, 15.1. FT-IR (KBr, $\left.v_{\max }, \mathrm{cm}^{-1}\right): 3061,2924,1746$, 1598, 1446, 1313, 1242, 1058, 908, 764, 700, 567.

Compound 3c: White solid, m.p. $95-96{ }^{\circ} \mathrm{C}$ (lit. ${ }^{13} 98^{\circ} \mathrm{C}$ ). ${ }^{1} \mathrm{H}$ NMR (400 MHz, DMSO- $\left.d_{6}\right)$ : $\delta \mathrm{H} 8.92$ (s, $1 \mathrm{H}, \mathrm{N}=\mathrm{CH}$ ), 8.07 (d, 2H, J 7.5 Hz, Ar-H), 7.82 (d, 2H, J 6.8 Hz, Ar-H), 7.73 (t, 1H, J 7.4 Hz, Ar-H), 7.52-7.62 (m, 5H, Ar-H). ${ }^{13} \mathrm{C}$ NMR (100 MHz, DMSO- $\left.d_{6}\right): \delta 171.8,156.7,134.2,133.7$, $133.4,131.8,130.2,129.8,129.7,128.9,128.8,128.5,128.4$. FT-IR $\left(\mathrm{KBr}, \mathrm{v}_{\max }, \mathrm{cm}^{-1}\right)$ : 3061, 2924, 1744, 1599, 1448, 1244, 1061, 864, 760, 704, 513.

Compound 3d: White solid, m.p. 55-56 ${ }^{\circ} \mathrm{C}$ (lit. ${ }^{21} 56-$ $57{ }^{\circ} \mathrm{C}$ ). ${ }^{1} \mathrm{H}$ NMR (400 MHz, DMSO- $d_{6}$ ): $\delta \mathrm{H} 8.08$ (d, 2H, J 7.4 $\mathrm{Hz}, \mathrm{Ar}-\mathrm{H}), 7.60$ (t, 1H, J 7.4 Hz, Ar-H), 7.48 (t, 2H, J 7.7 Hz, Ar-H), 2.69 (t, 2H, J 6.4 Hz, $\mathrm{CH}_{2}$ ), 2.49 (t, J $6.4 \mathrm{~Hz}, 2 \mathrm{H}, \mathrm{CH}_{2}$ ), 1.65-1.85 (m, 6H, $\left.\mathrm{CH}_{2}\right) .{ }^{13} \mathrm{C}$ NMR (100 MHz, DMSO- $\left.d_{6}\right): \delta$ 164.4, 164.1, 132.9, 129.8, 129.3, 129.1, 128.3, 128.1, 32.0, 26.9, 26.6, 25.7, 25.5. FT-IR ( KBr, $\left.v_{\max }, \mathrm{cm}^{-1}\right): 3066,2935$, 1738, 1637, 1450, 1263, 1062, 921, 864, 702.

Compound 3e: Colorless liquid (lit. colorless liquid ${ }^{22}$ ). ${ }^{1} \mathrm{H}$ NMR (400 MHz, DMSO- $d_{6}$ ): $\delta \mathrm{H} 8(\mathrm{~d}, 2 \mathrm{H}, J 7.4 \mathrm{~Hz}, \mathrm{Ar}-\mathrm{H}$ ), 7.68 (t, 1H, J 7.4 Hz, Ar-H), 7.55 (t, 2H, J 7.7 Hz, Ar-H), 2.08 (s, 3H, $\left.\mathrm{CH}_{3}\right), 2.03$ (s, 3H, $\left.\mathrm{CH}_{3}\right) .{ }^{13} \mathrm{C}$ NMR (100 MHz, DMSO$\left.d_{6}\right): \delta 164.5,163.8,133.1,129.4,129.2,128.4,21.9,16.9$. FT-IR $\left(\mathrm{KBr}, v_{\max }, \mathrm{cm}^{-1}\right)$ : 3068, 2926, 1741, 1627, 1450, 1373, 1274, 1247, 1085, 1062, 1022, 908, 864, 709.

Compound 3f: White solid, m.p. $143-145{ }^{\circ} \mathrm{C}$ (lit. $.^{23} 143-$ $\left.144{ }^{\circ} \mathrm{C}\right) .{ }^{1} \mathrm{H}$ NMR $\left(400 \mathrm{MHz}, \mathrm{DMSO}-d_{6}\right): \delta \mathrm{H} 8.95(\mathrm{~s}, 1 \mathrm{H}$, $\mathrm{N}=\mathrm{CH}), 8.06$ (d, 2H, J 7.6 Hz, Ar-H), 7.84 (d, 2H, J 8.1 Hz, Ar-H), 7.73 (t, 1H, J 7.2 Hz, Ar-H), 7.60 (t, 4H, J 7.9 Hz, ArH). ${ }^{13} \mathrm{C}$ NMR (100 MHz, DMSO- $\left.d_{6}\right): \delta 163.0,157.0,136.5$, $133.8,130.0,129.2,129.0,128.0$. FT-IR $\left(\mathrm{KBr}, v_{\max }, \mathrm{cm}^{-1}\right)$ : 3055, 1742, 1595, 1489, 1255, 1082, 934, 831, 702, 514.

Compound 3g: White solid, m.p. $69-70{ }^{\circ} \mathrm{C}$ (lit. ${ }^{24} 69$ $\left.70{ }^{\circ} \mathrm{C}\right) .{ }^{1} \mathrm{H}$ NMR (400 MHz, DMSO-d $)$ ): $\delta \mathrm{H} 7.96$ (d, 2H, $J 8$ $\mathrm{Hz}, \mathrm{Ar}-\mathrm{H}), 7.26$ (d, 2H, J $6 \mathrm{~Hz}, \mathrm{Ar}-\mathrm{H}), 2.68$ (t, 2H, $J 6 \mathrm{~Hz}$, $\mathrm{CH}_{2}$ ), 2.48 (t, 2H, J $6 \mathrm{~Hz}, \mathrm{CH}_{2}$ ), 2.43 (s, 3H, $\left.\mathrm{CH}_{3}\right), 1.63-1.84$ $\left(\mathrm{m}, 6 \mathrm{H}, \mathrm{CH}_{2}\right) .{ }^{13} \mathrm{C}$ NMR $\left(100 \mathrm{MHz}, \mathrm{DMSO}-d_{6}\right): \delta 169.3,164.3$, 144.5, 143.8, 130.2, 129.6, 129.1, 126.6, 32.2, 27.1, 26.7, 25.8. FT-IR $\left(\mathrm{KBr}, v_{\max }, \mathrm{cm}^{-1}\right): 2933,1730,1606,1448,1267,1169$, 1076, 918, 833, 750, 478.

Compound 3h: White solid, m.p. $117-119{ }^{\circ} \mathrm{C}$ (lit. ${ }^{25} 116-$ $\left.118{ }^{\circ} \mathrm{C}\right) .{ }^{1} \mathrm{H}$ NMR (400 MHz, DMSO- $\left.d_{6}\right): \delta \mathrm{H} 7.98(\mathrm{~d}, 2 \mathrm{H}, J 8$ $\mathrm{Hz}, \mathrm{Ar}-\mathrm{H}), 7.82$ (d, 2H, J 8 Hz, Ar-H), 7.48-7.56 (m, 3H, Ar$\mathrm{H}), 7.40$ (d, 2H, J $8 \mathrm{~Hz}, \mathrm{Ar}-\mathrm{H}), 2.51\left(\mathrm{~s}, 3 \mathrm{H}, \mathrm{CH}_{3}\right), 2.41$ (s, 3H, $\left.\mathrm{CH}_{3}\right) .{ }^{13} \mathrm{C}$ NMR $\left(100 \mathrm{MHz}, \mathrm{DMSO}-d_{6}\right): \delta 163.8,162.9,134.5$, $130.7,129.5,129.3,128.7,126.9,125.8,21.2,14.3$. FT-IR $\left(\mathrm{KBr}, \mathrm{v}_{\max }, \mathrm{cm}^{-1}\right): 3032,2922,1732,1607,1443,1312,1250$, 1068, 899, 748, 696, 567.

Compound 3i: White solid, m.p. 91-93 ${ }^{\circ} \mathrm{C} .{ }^{1} \mathrm{H}$ NMR (400 MHz, DMSO- $d_{6}$ ): $\delta \mathrm{H} 8.93$ (s, 1H, N=CH), 7.95 (d, 2H, $J 8.1$ $\mathrm{Hz}, \mathrm{Ar}-\mathrm{H}), 7.83$ (d, 2H, J 8.4 Hz, Ar-H), 7.61 (d, 2H, J 8.4 Hz, Ar-H), 7.40 (d, 2H, J $8.0 \mathrm{~Hz}, \mathrm{Ar}-\mathrm{H}), 2.41$ (s, 3H, $\left.\mathrm{CH}_{3}\right) .{ }^{13} \mathrm{C}$ NMR (100 MHz, DMSO- $\left.d_{6}\right)$ : $\delta 163.0,156.8,144.4,136.4$, 134.1, 129.9, 129.5, 129.3, 129.1, 125.2, 21.2. FT-IR (KBr, $\left.V_{\max }, \mathrm{cm}^{-1}\right): 3031,2924,1737,1612,1489,1261,1080,937$, 817, 740, 513.

Compound 3j: Light yellow solid, m.p. $110-111^{\circ} \mathrm{C}$ (lit. ${ }^{26}$ 111-112 ${ }^{\circ} \mathrm{C}$ ). ${ }^{1} \mathrm{H}$ NMR (400 MHz, DMSO- $\left.d_{6}\right)$ : $\delta \mathrm{H} 8.35(\mathrm{~d}$, $2 \mathrm{H}, J 8.8 \mathrm{~Hz}, \mathrm{Ar}-\mathrm{H}), 8.23$ (d, 2H, J 8.8 Hz, Ar-H), 2.66 (t, 2H, $\left.J 6.2 \mathrm{~Hz}, \mathrm{CH}_{2}\right), 2.38$ (t, 2H, J $\left.6 \mathrm{~Hz}, \mathrm{CH}_{2}\right), 1.60-1.74(\mathrm{~m}, 6 \mathrm{H}$, $\left.\mathrm{CH}_{2}\right) .{ }^{13} \mathrm{C}$ NMR (100 MHz, DMSO- $\left.d_{6}\right): \delta 170.7,161.8,150.3$, 134.3, 130.6, 123.9, 31.2, 26.5, 26.3, 25.4, 24.6. FT-IR (KBr, $\left.v_{\max }, \mathrm{cm}^{-1}\right): 3109,2949,1730,1629,1525,1346,1267,1093$, 854,711 .

Compound 3k: Light yellow solid, m.p. $197-198{ }^{\circ} \mathrm{C} .{ }^{1} \mathrm{H}$ NMR (400 MHz, DMSO-d $)_{6}$ : $\delta \mathrm{H} 9.00(\mathrm{~s}, 1 \mathrm{H}, \mathrm{N}=\mathrm{CH}), 8.41$ (d, 2H, J $8.8 \mathrm{~Hz}$, Ar-H), 8.29 (d, 2H, J $8.8 \mathrm{~Hz}, \mathrm{Ar}-\mathrm{H}), 7.85$ (d, $2 \mathrm{H}, J 8.8 \mathrm{~Hz}, \mathrm{Ar}-\mathrm{H}), 7.62$ (d, $2 \mathrm{H}, J 8.4 \mathrm{~Hz}, \mathrm{Ar}-\mathrm{H}) .{ }^{13} \mathrm{C}$ NMR $\left(100 \mathrm{MHz}, \mathrm{DMSO}-d_{6}\right): \delta 161.7,157.8,150.5,136.8,133.6$, 130.8, 130, 129.3, 128.7, 124.0. FT-IR (KBr, $\left.v_{\max }, \mathrm{cm}^{-1}\right): 3084$, 1757, 1610, 1527, 1346, 1257, 1082, 931, 831, 707, 518.

\section{RESULTS AND DISCUSSION}

Our optimization of the reaction conditions involved us studying the oxime esterification of benzoic acid with benzophenone oxime. Initially, when benzoic acid was treated with 1 equiv of benzophenone oxime, 1 equiv of 2 - $(1 \mathrm{H}$-benzotriazole-1-yl)-1,1,3,3-tetramethyluronium tetrafluoroborate and 1 equiv of triethylamine at room temperature for $0.5 \mathrm{~h}$ in acetonitrile, $71.6 \%$ of product was obtained. It is worth pointing out that lengthening the reaction time to $5 \mathrm{~h}$ did not affect the yield of the reaction. We attempted to use DIPEA, DBU, methylamine, or pyridine as the base instead of triethylamine, nevertheless, they gave a lower yield. Dichloromethane and tetrahydrofuran were also evaluated as reaction solvents and the yields were 62.1 and $62.7 \%$, respectively. However, acetonitrile was the most effective solvent. Further, the influence of 2-(1H-benzotriazole-1-yl)-1,1,3,3-tetramethyluronium tetrafluoroborate and triethylamine in reaction of benzoic acid and cyclohexanone oxime was also investigated and the results are recorded in Table- 1 . Table- 1 reveals, in the absence of 2-(1H-benzotriazole-1-yl)-1,1,3,3-tetramethyluronium tetrafluoroborate, no product was observed (Table-1, Entry-1). Therefore, the results indicated that the best conditions for obtaining oxime ester entailed us using 1 equiv of 
benzoic acid, 1 equiv of benzophenone oxime, 1.1 equiv of 2(1H-benzotriazole-1-yl)-1,1,3,3-tetramethyluronium tetrafluoroborate and 2 equiv of triethylamine at room temperature for $0.5 \mathrm{~h}$ in acetonitrile and benzophenone $O$-benzoyloxime was obtained in $89.5 \%$ yield (Table-1, Entry-11).

\begin{tabular}{|c|c|c|c|}
\hline \multicolumn{4}{|c|}{$\begin{array}{c}\text { Table-1 } \\
\text { OPTIMIZATION OF 2-( } 1 H \text {-BENZOTRIAZOLE-1-YL)-1,1,3,3 } \\
\text { TETRAMETHYLURONIUM TETRAFLUOROBORATE } \\
\text { (TBTU) LOADING AND TRIETHYLAMINE (TEA) } \\
\text { AMOUNT IN THE REACTION OF BENZOIC ACID } \\
\text { WITH BENZOPHENONE OXIME }{ }^{\mathrm{a}}\end{array}$} \\
\hline Entry & TBTU (equiv.) & TEA (equiv.) & Yield (\%) \\
\hline 1 & 0.0 & 1.0 & - \\
\hline 2 & 0.5 & 1.0 & 61.7 \\
\hline 3 & 0.75 & 1.0 & 69.6 \\
\hline 4 & 1.0 & 1.0 & 71.6 \\
\hline 5 & 1.1 & 1.0 & 72.2 \\
\hline 6 & 1.2 & 1.0 & 72.2 \\
\hline 7 & 1.1 & 1.2 & 74.6 \\
\hline 8 & 1.1 & 1.4 & 79.5 \\
\hline 9 & 1.1 & 1.6 & 82.3 \\
\hline 10 & 1.1 & 1.8 & 86.8 \\
\hline 11 & 1.1 & 2.0 & 89.5 \\
\hline 12 & 1.1 & 2.2 & 89.5 \\
\hline
\end{tabular}

${ }^{a}$ Reaction conditions: Reactions carried out using benzoic acid (1.0 equiv) and benzophenone oxime (1.0 equiv) in acetonitrile at room temperature for $0.5 \mathrm{~h}$.

Subsequently, we investigated the scope of the reaction substrates under the optimized conditions, as shown in Table- 2 . From Table-2, we can see, by this method, that the aromatic carboxylic acids gave satisfactory results (Table-2, Entries 111). Unfortunately, when acetic acid, chloroacetic acid or hexanoic acid was used in this reaction, no corresponding product was isolated (Table-2, Entries-12-14). Benzoic acid reacted with a series of oximes, of which the non-cyclic aliphatic oxime gave the lowest yield (Table-2, Entry-5). We were also interested to study the electronic effect of substitutions on aromatic ring of benzoic acids. The substitution of an electron-donating methyl group and the substitution of an electron-withdrawing nitro group did not affect the yields (Table-2, Entry-7-11).

\begin{tabular}{|c|c|c|c|c|c|c|}
\hline & $\begin{array}{r}\text { REACTIC } \\
\text { OXIN }\end{array}$ & $\begin{array}{r}\text { TA } \\
\text { NS OF CA } \\
\text { ES AT RO }\end{array}$ & $\begin{array}{l}\text { LE } 2 \\
\text { 3OXYL } \\
\text { M TEM }\end{array}$ & $\begin{array}{l}\text { C ACIDS } \\
\text { ERATUR }\end{array}$ & $\begin{array}{l}\text { AND } \\
\text { E }\end{array}$ & \\
\hline Entry & $\mathrm{R}^{1}$ & $\mathrm{R}^{2}$ & $\mathrm{R}^{3}$ & Product & $\begin{array}{l}\text { Time } \\
\text { (h) }\end{array}$ & $\begin{array}{l}\text { Yield } \\
(\%)\end{array}$ \\
\hline 1 & $\mathrm{C}_{6} \mathrm{H}_{5}$ & $\mathrm{C}_{6} \mathrm{H}_{5}$ & $\mathrm{C}_{6} \mathrm{H}_{5}$ & 3a & 0.5 & 89.5 \\
\hline 2 & $\mathrm{C}_{6} \mathrm{H}_{5}$ & $\mathrm{C}_{6} \mathrm{H}_{5}$ & $\mathrm{CH}_{3}$ & $3 \mathbf{b}$ & 0.5 & 88.6 \\
\hline 3 & $\mathrm{C}_{6} \mathrm{H}_{5}$ & $\mathrm{C}_{6} \mathrm{H}_{5}$ & $\mathrm{H}$ & $3 c$ & 0.5 & 83.6 \\
\hline 4 & $\mathrm{C}_{6} \mathrm{H}_{5}$ & $\left(\mathrm{CH}_{2}\right)_{5}$ & & 3d & 0.5 & 85.2 \\
\hline 5 & $\mathrm{C}_{6} \mathrm{H}_{5}$ & $\mathrm{CH}_{3}$ & $\mathrm{CH}_{3}$ & $3 e$ & 0.5 & 65.5 \\
\hline 6 & $\mathrm{C}_{6} \mathrm{H}_{5}$ & $4-\mathrm{ClC}_{6} \mathrm{H}_{5}$ & $\mathrm{H}$ & $3 f$ & 0.5 & 92.1 \\
\hline 7 & 4- $\mathrm{CH}_{3} \mathrm{C}_{6} \mathrm{H}_{4}$ & $\left(\mathrm{CH}_{2}\right)_{5}$ & & $3 g$ & 0.5 & 86.6 \\
\hline 8 & 4- $\mathrm{CH}_{3} \mathrm{C}_{6} \mathrm{H}_{4}$ & $\mathrm{C}_{6} \mathrm{H}_{5}$ & $\mathrm{CH}_{3}$ & $3 \mathrm{~h}$ & 0.5 & 87.4 \\
\hline 9 & 4- $\mathrm{CH}_{3} \mathrm{C}_{6} \mathrm{H}_{4}$ & 4- $\mathrm{ClC}_{6} \mathrm{H}_{5}$ & $\mathrm{H}$ & $3 \mathbf{i}$ & 0.5 & 90.8 \\
\hline 10 & $4-\mathrm{NO}_{2} \mathrm{C}_{6} \mathrm{H}_{4}$ & $\left(\mathrm{CH}_{2}\right)_{5}$ & & $3 \mathbf{j}$ & 0.5 & 87.0 \\
\hline 11 & $4-\mathrm{NO}_{2} \mathrm{C}_{6} \mathrm{H}_{4}$ & $4-\mathrm{ClC}_{6} \mathrm{H}_{5}$ & $\mathrm{H}$ & $3 k$ & 0.5 & 77.5 \\
\hline 12 & $\mathrm{CH}_{3}$ & $\mathrm{C}_{6} \mathrm{H}_{5}$ & $\mathrm{C}_{6} \mathrm{H}_{5}$ & 31 & 5.0 & - \\
\hline 13 & $\mathrm{Cl}-\mathrm{CH}_{2}$ & $\mathrm{C}_{6} \mathrm{H}_{5}$ & $\mathrm{C}_{6} \mathrm{H}_{5}$ & $3 \mathrm{~m}$ & 5.0 & - \\
\hline 14 & $\mathrm{C}_{5} \mathrm{H}_{11}$ & $\mathrm{C}_{6} \mathrm{H}_{5}$ & $\mathrm{C}_{6} \mathrm{H}_{5}$ & $3 n$ & 5.0 & - \\
\hline
\end{tabular}

We also investigated the mechanism of the reaction (Scheme-II). According to the literature ${ }^{27,28}$, the reaction mechanism for oxime ester bond formation through 2-(1Hbenzotriazole-1-yl)-1,1,3,3-tetramethyluronium tetrafluoroborate activation mechanism has been proposed for this coupling reaction. The proposed mechanism may consist of the following steps: Firstly, addition of the carboxylate anion to 2-(1H-benzotriazole-1-yl)-1,1,3,3-tetramethyluronium tetrafluoroborate. Secondly, decomposition of the resulting intermediate acyloxy-aminium/uronium salt. After that, addition of the released anion to the carbonyl center, followed by loss of water-miscible tetramethylurea. Finally, oximes react with the activated carbonyl group to give the corresponding oxime esters and 1-hydroxybenzotriazole (HOBt) as a watersoluble by-product.

Geometric optimization of the products was carried out at the b3lyp/6-311G(d,p) level along with analytic vibrational frequency calculations with the Gaussian-03 software package ${ }^{29}$. The frequencies were scaled by a factor of 0.967 . The calculated vibrational frequencies are consistent with the experimental infrared data.

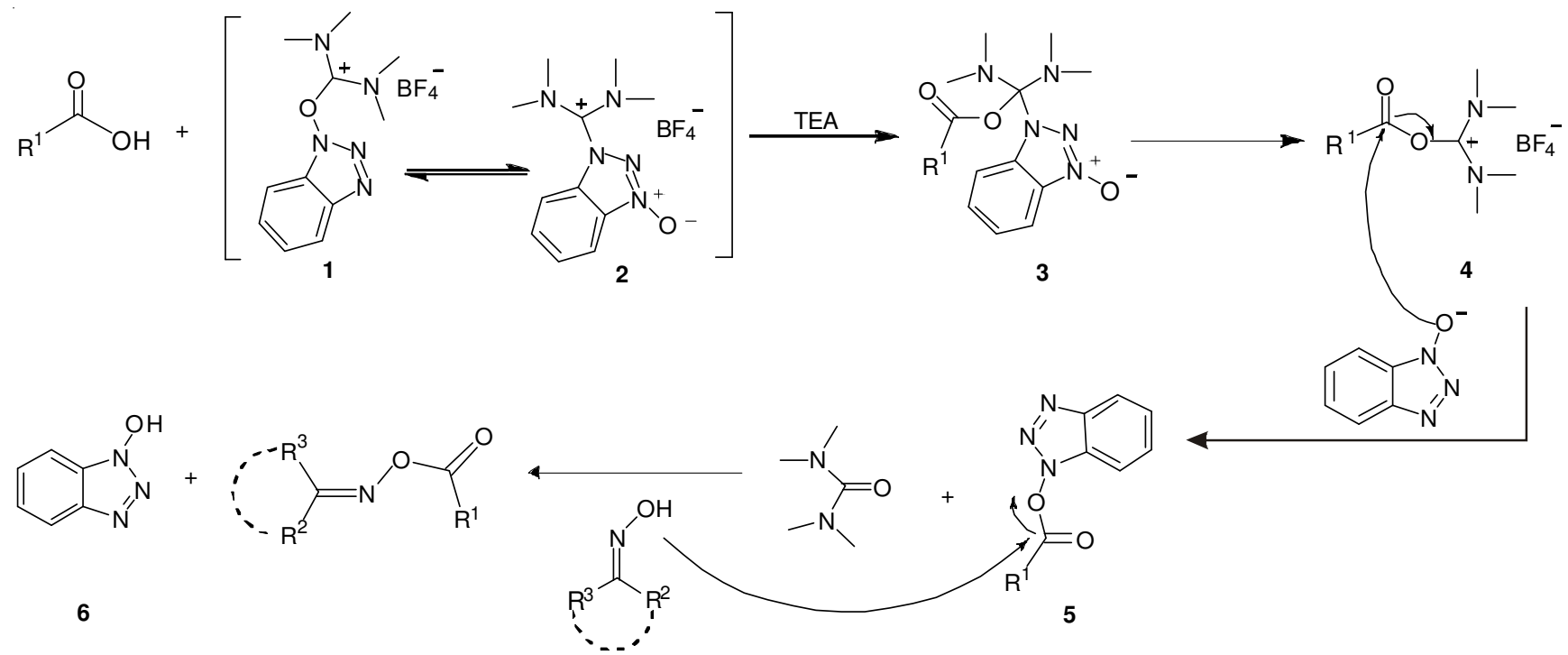

Scheme-II: Proposed reaction mechanism for oxime ester bond formation through 2-(1H-benzotriazole-1-yl)-1,1,3,3-tetramethyluronium tetrafluoroborate activation 


\section{Conclusion}

In summary, a practical method for the synthesis of carboxylic acid oxime ester using commercially available and inexpensive carboxylic acid, oxime ester and 2-( $1 \mathrm{H}$-benzotriazole-1-yl)-1,1,3,3-tetramethyluronium tetrafluoroborate in acetonitrile at room temperature under mild conditions has been developed. Additionally, the novel method offers several advantages, such as the generation of water-soluble and lowtoxicity by-products, ease of product separation and short reaction time.

\section{ACKNOWLEDGEMENTS}

This work was supported by the Natural Science Foundation of Shandong Province, China (No. ZR2012BM004) and the Project of Shandong Province Higher Educational Science and Technology Program (No. J12LD07).

\section{REFERENCES}

1. A. Karakurt, M.A. Alagoz, B. Sayoglu, U. Calis and S. Dalkara, Eur. J. Med. Chem., 57, 275 (2012).

2. G. Surkau, K.J. Böhm, K. Müller and H. Prinz, Eur. J. Med. Chem., 45 3354 (2010).

3. Y.J. Cui, X.P. Rao, S.B. Shang, S. Jie and Y.Q. Gao, Lett. Drug Des. Discov., 10, 102 (2013).

4. H.Q. Zhao, M.J. Zhou, L.F. Duan, W. Wang, J.J. Zhang, D.Q. Wang and X.M. Liang, Molecules, 18, 3615 (2013).

5. C.D. Magnusson and G.G. Haraldsson, Chem. Phys. Lipids, 165, 712 (2012).

6. R. Sun, Y. Li, M. Lü, L. Xiong and Q. Wang, Bioorg. Med. Chem. Lett., 20, 4693 (2010).

7. N.J. Turro, Y.J. Li, S. Jockusch, Y. Hagiwara, M. Okazaki, R.A. Mesch D.I. Schuster and C.G. Willson, J. Org. Chem., 78, 1735 (2013).

8. J. Xu, G.L. Ma, K.M. Wang, J.M. Gu, S. Jiang and J. Nie, J. Appl. Polym. Sci., 123, 725 (2012).

9. S.S.P. Chou, J.C. Juan, S.C. Tsay, K.P. Huang and J.R. Hwu, Molecules, 17, 3370 (2012).

10. C. Dworak and R. Liska, J. Polym. Sci. A, 48, 5865 (2010).

11. S.B. Liu, Y. Yu and L.S. Liebeskind, Org. Lett., 9, 1947 (2007).

12. D.J. Ritson, R.J. Cox and J. Berge, Org. Biomol. Chem., 2, 1921 (2004).
13. S.K. Kundu, M. Rahman, P. Dhara, A. Hajra and A. Majee, Synth. Commun., 42, 1848 (2012).

14. S. Nakaiida, S. Kato, O. Niyomura, M. Ishida, F. Ando and J. Koketsu, Phosphorus Sulfur Rel. Elem., 185, 930 (2010).

15. B. Kongkathip, S. Akkarasamiyo, K. Hasitapan, P. Sittikul, N. Boonyalai and N. Kongkathip, Eur. J. Med. Chem., 60, 271 (2013).

16. H. Chen, X.F. Xu, L. Liu, G. Tang and Y.F. Zhao, RSC Adv., 3, 16247 (2013).

17. R. Subirós-Funosas, L. Nieto-Rodriguez, K.J. Jensen and F. Albericio, J. Pept. Sci., 19, 408 (2013).

18. S. Balalaie, M. Mahdidoust and R. Eshaghi-Najafabadi, Chin. J. Chem., 26, 1141 (2008).

19. S. Balalaie, M. Mahdidoust and R. Eshaghi-Najafabadi, J. Iran. Chem. Soc., 4, 364 (2007).

20. M.Z.A. Badr, M.M. Aly, S.A. Mahgoub, A.M. Fahmy and A.A. Atallah, Bull. Chem. Soc. Jpn., 61, 1779 (1988).

21. E.A. Dikusar and N.A. Zhukovskaya, Russ. J. Org. Chem., 44, 1389 (2008).

22. S. Nakaiida, S. Kato, O. Niyomura, M. Ishida, F. Ando and J. Koketsu, Phosphorus Sulfur Rel. Elem., 185, 930 (2010).

23. C.R. Hauser and G. Vermillion, J. Am. Chem. Soc., 63, 1224 (1941).

24. A.R. Katritzky, A.A.A. Abdel-Fattah, A.V. Gromova, R. Witek and P.J. Steel, J. Org. Chem., 70, 9211 (2005).

25. W. Renfrow, J. White, R. Wolf and W. Bohl, J. Org. Chem., 33, 150 (1968).

26. L. Gass and F.W. Bope, J. Am. Pharm. Assoc., 48, 186 (1959).

27. J.K. Twibanire and T.B. Grindley, Org. Lett., 13, 2988 (2011).

28. B. Movassagh, S. Balalaie and P. Shaygan, ARKIVOC, 47 (2007).

29. M.J. Frisch, G.W. Trucks, H.B. Schlegel, G.E. Scuseria, M.A. Robb, J.R. Cheeseman, J.A. Montgomery Jr., T. Vreven, K.N. Kudin, J.C. Burant, J.M. Millam, S.S. Iyengar, J. Tomasi, V. Barone, B. Mennucci, M. Cossi, G. Scalmani, N. Rega, G.A. Petersson, H. Nakatsuji, M. Hada, M. Ehara, K. Toyota, R. Fukuda, J. Hasegawa, M. Ishida, T. Nakajima, Y. Honda, O. Kitao, H. Nakai, M. Klene, X. Li, J.E. Knox, H.P. Hratchian, J.B. Cross, V. Bakken, C. Adamo, J. Jaramillo, R. Gomperts, R.E. Stratmann, O. Yazyev, A.J. Austin, R. Cammi, C. Pomelli, J.W. Ochterski, P.Y. Ayala, K. Morokuma, G.A. Voth, P. Salvador, J.J. Dannenberg, V.G. Zakrzewski, S. Dapprich, A.D. Daniels, M.C. Daniels, O. Farkas, D.K. Malick, A.D. Rabuck, K. Raghavachari, J.B. Foresman, J.V. Ortiz, Q. Cui, A.G. Baboul, S. Clifford, J. Cioslowski, B.B. Stefanov, G. Liu, A. Liashenko, P. Piskorz, I. Komaromi, R.L. Martin, D.J. Fox, T. Keith and M.A. Al-Laham, C.Y. Peng, A. Nanayakkara, M. Challacombe, P.M.W. Gill, B. Johnson, W. Chen, M.W. Wong, C. Gonzalez, J.A. Pople, Gaussian 03 (Revision A.1), Gaussian, Inc., Pittsburgh, PA (2004). 